\title{
Oscillation resonances and anisotropic damping of the motion of acoustically levitated droplets in single-axis acoustic levitators
}

Cite as: Appl. Phys. Lett. 115, 053702 (2019); doi: 10.1063/1.5112109

Submitted: 2 June 2019 - Accepted: 12 July 2019 .

Published Online: 1 August 2019

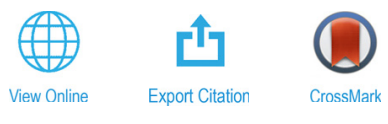

Soichiro Tsujino, $^{1, a)}$ (D) Yohei Sato, ${ }^{2}$ (D) Yasushi Takeda, ${ }^{3}$ and Takashi Tomizaki ${ }^{1}$ (iD

\author{
AFFILIATIONS \\ ${ }^{7}$ Photon Science Division, Paul Scherrer Institut, Villigen PSI 5232, Switzerland \\ ${ }^{2}$ Nuclear Energy and Safety Research Division, Paul Scherrer Institut, Villigen PSI 5232, Switzerland \\ ${ }^{3}$ Institute for Food, Nutrition and Health, ETH Zürich, 8092 Zürich, Switzerland \\ a) Electronic mail: soichiro.tsujino@psi.ch
}

\begin{abstract}
The positional stability of acoustically levitated droplets in air and the settling time of droplet positions on loading are important for precision applications of acoustic levitation. We therefore study their dependence on ultrasound pressure for droplets with diameters 0.01-0.2 times the acoustic wavelength in a single-axis acoustic levitator operating at $39 \mathrm{kHz}$. We find that the observed resonance frequencies agree well with theory. However, the damping coefficients of the oscillations exhibit large anisotropy, and their dependence on the droplet size deviates greatly from the behavior expected from the Stokes coefficient. These results suggest that acoustic streaming plays an important role in the motion of acoustically levitated droplets.
\end{abstract}

Published under license by AIP Publishing. https://doi.org/10.1063/1.5112109

\begin{abstract}
Understanding the motion of an acoustically levitated droplet is important to achieve the high positional stability required for precision applications of acoustic levitation such as in X-ray diffraction experiments for protein crystallography. ${ }^{1-3}$ The acoustic radiation force ${ }^{4,5}$ of an ultrasonic standing wave acting on particles and droplets in ambient air has been extensively studied, and the theory developed for small spherical particles in an inviscid fluid has been utilized to design and analyze acoustic levitation in the context of various applications. ${ }^{6-19}$ Recently, we proposed to use acoustic levitation in protein crystallography to realize a system for fully automated and high-throughput $\mathrm{X}$-ray diffraction experiments at room temperatures at synchrotron facilities. ${ }^{1-3}$ For this to be possible, one of the important requirements is that the positional stability of the levitated droplets must be better than $0.05-0.1 \mathrm{~mm}$ (i.e., smaller than the size of crystals contained in the droplets) for droplets with diameter $d$ in the range of $0.5-1 \mathrm{~mm}$ or smaller. This is to secure a high hit-rate of the X-ray beam on the crystals and to maximize the signal-to-noise ratio of the diffraction signals by minimizing the reduction of the diffraction signal due to water absorption. These experiments have so far been conducted using ultrasound with a frequency of $\sim 39 \mathrm{kHz}$ (corresponding to a wavelength $\lambda$ of $\sim 8.9 \mathrm{~mm}$ in ambient air at $20^{\circ} \mathrm{C}$ ). The motion of acoustically levitated droplets in ambient air has been extensively studied. ${ }^{2}$
\end{abstract}

However, investigations of the resonance oscillation characteristics of levitated droplets together with their damping behavior are rare, especially for droplets with $d / \lambda$ much smaller than 0.1 . In this letter, we therefore study the influence of the droplet size and ultrasound pressure on the oscillation characteristics of levitated droplets in a single-axis levitator and their impact on the positional stability of levitated droplets for $d / \lambda$ in the range of $0.01-0.2$.

To study the resonance characteristics of the oscillatory motion of levitated water droplets, we apply a small amount of amplitude modulation (AM) of the ultrasound pressure amplitude $P_{\mathrm{ac}}$ at low frequencies $\nu$ and record the droplet images using a highspeed camera. When $\nu$ is close to the axial resonance frequency $\omega_{z 0}$, the center-of-mass motion of a levitated droplet in the axial direction under AM can be written as (see Appendix A1, supplementary material)

$$
\frac{d^{2}}{d t^{2}}\left(z-z_{0}\right)+\omega_{z 0}^{2}\left(z-z_{0}\right)=\alpha_{d}+2 \varepsilon g \cos \nu t
$$

where $\varepsilon(\ll 1)$ is the fractional amount of $P_{\text {ac }}$ modulation, $g=9.8 \mathrm{~m} / \mathrm{s}^{2}$ is the acceleration due to gravity, and $z_{0}$ is the equilibrium position [see Eq. (S23) in the supplementary material]. $\alpha_{d}$ is the acceleration by the drag force ${ }^{26}$ 


$$
\alpha_{d}=-\gamma\left[\frac{d}{d t}\left(z-z_{0}\right)-u_{f, z}\right],
$$

where $\gamma$ is the damping coefficient. We have introduced here the $(z$ component of the) average air flow velocity, $u_{f, z}$, which is zero for large droplets, but may take a finite value for small droplets. In the absence of ultrasound, $\gamma$ for a spherical droplet of diameter $d$ at low velocities (or with the Reynolds number below $\sim 1$ ) is given by the ratio $\gamma_{S}$ of the Stokes coefficient $6 \pi \mu(d / 2)$ to the droplet mass $(4 \pi / 3) \rho(d / 2)^{3}$ (where $\mu=1.85 \times 10^{-5} \mathrm{~Pa}$ s is the viscosity of ambient air at $20^{\circ} \mathrm{C}$ and $\rho=10^{3} \mathrm{~kg} / \mathrm{m}^{3}$ is the density of water). ${ }^{26}$ Because of the small value of $\varepsilon$ and the condition $\nu \sim \omega_{z 0}$, the nonlinearity ${ }^{21,27}$ of the acoustic radiation force has been neglected, as it has terms that vary with the frequency equal to and higher than $2 \nu$. When the levitator axis is tilted by a small angle $\phi$ from the vertical direction, droplet motion in the radial direction can be excited when $\nu$ is close to the angular frequency $\omega_{r 0}$ of the radial resonance, and the motion is described by a similar equation, but with $g$ replaced by $g \sin \phi$, the equilibrium position replaced by $r_{0} \sim 0$, and $u_{f, z}$ replaced by $u_{f, r}$.

We find that the relationship between the ultrasound pressure and the axial and radial resonance frequencies agrees well with theory, but an anisotropic and anomalous $d$ dependence is observed for the damping coefficient, the values of which deviate strongly from those expected from the Stokes coefficient. We discuss the consequences for the positional stability of levitated droplets, as well as the influence of other effects such as acoustic streaming on small levitated droplets.

We used a single-axis acoustic levitator consisting of an ultrasonic transducer (a bolt-clamped Langevin-type piezoelectric transducer) and a concave mirror reflector [Fig. 1(a)] operating at an ultrasound frequency $f_{\mathrm{ac}}$ of $\sim 39 \mathrm{kHz}$. $^{3}$ The distribution of the ultrasound pressure [Fig. 1(b)] and the time-averaged pressure [Fig. 1(c)] of the acoustic standing wave in the levitator was characterized by schlieren imaging ${ }^{3,28}$ combined with a high-speed camera (Photron AX100) or a CCD camera. From these images, we found that the radial spread of the standing wave at the middle node was described well by $\exp \left(-r^{2} / s^{2}\right)$ with $s=7.24 \pm 0.11 \mathrm{~mm}$ (see Appendix A2, supplementary material) when the levitator cavity was adjusted to the fifth resonance, i.e., the distance between the transducer end and the mirror reflector was approximately equal to $n \lambda / 2$ with $n=5$, where $\lambda=c_{0} / f_{\text {ac }}$ (where $c_{0}=343 \mathrm{~m} / \mathrm{s}$ is the speed of sound in ambient air at $20^{\circ} \mathrm{C}$ ). The ultrasonic velocity amplitude $V_{\mathrm{ac}}$ at the middle node and the corresponding ultrasonic pressure $P_{\mathrm{ac}}=\rho_{0} c_{0} V_{\mathrm{ac}}$ (where $\rho_{0}=1.204 \mathrm{~kg} / \mathrm{m}^{3}$ is the density of air) were evaluated from the voltage signal $v_{p}$ at $f_{\mathrm{ac}}$ of a piezosensor attached to the mirror reflector. To obtain the constant of proportionality between $v_{p}$ and $P_{\mathrm{ac}}$, we measured the threshold sensor output $v_{p, 0}$ to levitate a water droplet (of density $\rho=10^{3} \mathrm{~g} / \mathrm{m}^{3}$ ) against gravity, ${ }^{29}$ and identified $v_{p} / v_{p, 0}$ with the ratio $P_{\mathrm{ac}} / P_{\mathrm{ac}, 0}$. We used the value of $v_{p, 0}$ measured when $d$ was equal to $0.7 \mathrm{~mm}$, since no change in $v_{p, 0}$ was detected for smaller droplets. No influence of droplet loading on $v_{p}$ or on the air-cavity resonance of the levitator was observed for the droplets with $d$ below $\sim 1 \mathrm{~mm}$ that were mainly studied in the present work (there was a noticeable effect, however, for droplets with $d$ larger than $\sim 2 \mathrm{~mm}$ ). We calculated $P_{\mathrm{ac}, 0}=1.35 \mathrm{kPa}$-rms for $f_{\mathrm{ac}}=39 \mathrm{kHz}$ from the theory outlined in Appendix A1 (supplementary material). In the experiments described below, the droplet shape was to a good approximation an oblate spheroid with $d_{z} / d_{x}$ between 0.8 and 1 , where $d_{x}$ and $d_{z}$ are the droplet diameters in the radial and axial directions, respectively. Therefore, we defined $d$ as $\left(d_{x}^{2} d_{z}\right)^{1 / 3}$, which is the diameter of a sphere with the same volume.

By finding the relationship between the AM frequency $\nu$ and the droplet oscillations, we obtained the resonance frequencies at various ultrasound pressures. In addition, we measured the oscillation decay on termination of the forced oscillations (induced by AM) and evaluated $\gamma$. To find the relationship between the droplet size and positional stability, we recorded the droplet images using a high-speed camera or a CCD camera at a constant $P_{\mathrm{ac}}$. From this relationship, we determined the evolution of the center-of-mass position $\left(x_{c}, z_{c}\right)$ in the axial and radial directions as a function of $d$. We also evaluated the positional fluctuations $\Delta x$ and $\Delta z$ as one-half of the differences between successive values of $x_{c}$ and $z_{c}$. During these experiments, we enclosed the levitator cavity in plastic film (forming an approximately $\sim 30 \mathrm{~cm}$ cube) to avoid any influence of ambient air disturbances on droplet motion. The ambient air was kept at $\sim 20^{\circ} \mathrm{C}$ and the relative humidity was in the range $20 \%-30 \%$.

Figures 2(a) and 2(b) show the resonance frequency characteristics for the radial and axial oscillations, respectively, measured at four different values of $P_{\mathrm{ac}}$. When $P_{\mathrm{ac}} / P_{\mathrm{ac}, 0} \sim 2.5$, the radial resonance frequency $f_{r 0}\left(=\omega_{r 0} / 2 \pi\right)$ was equal to $10 \mathrm{~Hz}$, and the axial resonance frequency $f_{z 0}\left(=\omega_{z 0} / 2 \pi\right)$ was equal to $42 \mathrm{~Hz}$. As shown in the insets, $f_{z 0}$ and $f_{r 0}$ were proportional to $P_{\mathrm{ac}}$ in the measured pressure range.
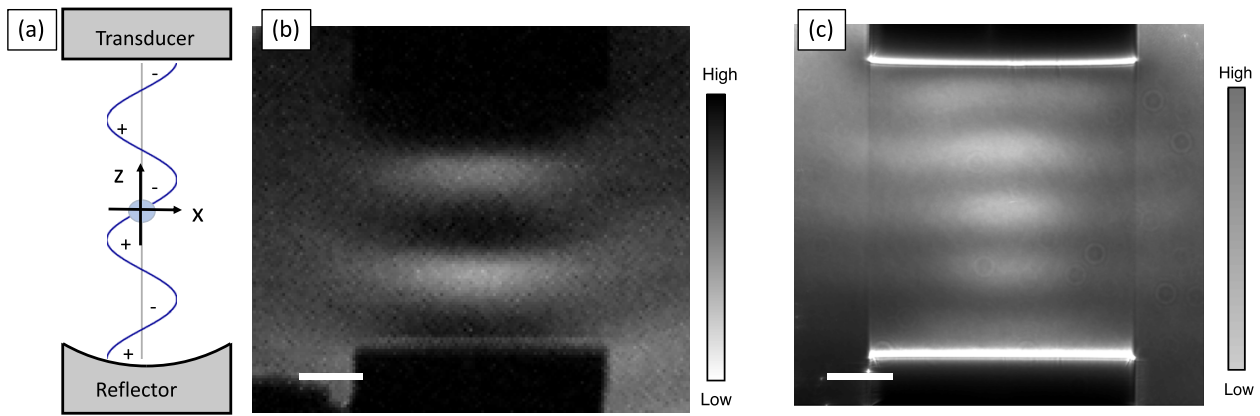

FIG. 1. (a) Schematic of the single-axis acoustic levitator adjusted to the fifth resonance. (b) and (c) are snapshots of the ultrasonic acoustic field of the $39 \mathrm{kHz}$ levitator (no levitated droplets) for (b) the instantaneous ultrasound pressure (at a phase when the pressure peak value was maximum below the middle pressure node) and (c) the timeaveraged pressure. The high- and low-pressure regions are dark and light, respectively. The scale bars are $5 \mathrm{~mm}$. The ultrasound frequency and the rms ultrasound pressure amplitude were $39.92 \mathrm{kHz}$ and $2.1 \mathrm{kPa}$ in (b) and $39.033 \mathrm{~Hz}$ and $2.3 \mathrm{kPa}$ in (c). 
(a)

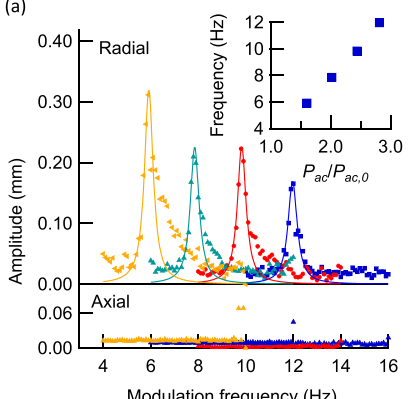

(b)

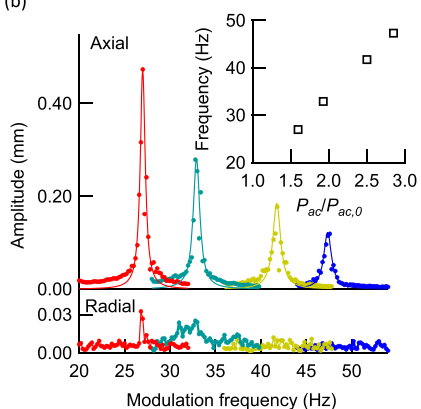

FIG. 2. Resonance characteristics of levitated droplet motion measured by amplitude modulation (AM) of the ultrasonic pressure $P_{\text {ac. }}$ (a) and (b), respectively, show the excitation of the radial and axial resonances observed by $1 \% \mathrm{AM}$ at different values of $P_{\text {ac }}$. The insets show the peak frequencies as functions of the ultrasonic pressure $P_{\text {ac. }}$. The curves were fitted by a Lorentzian. (a) was measured for $d$ between 1.35 and $1.72 \mathrm{~mm}$, and (b) for $d$ between 0.80 and $1.00 \mathrm{~mm}$.

Figure 3(a) compares the relationships between $P_{\mathrm{ac}}$ and the axial and radial resonance frequencies with theoretical results based on an approach of Gorkov. ${ }^{5}$ In the theoretical analysis, we included the radial spread of the ultrasound pressure evaluated from the schlieren image to the lowest order (see Appendix A1, supplementary material). The solid curves, which were calculated from the theory assuming $s=7.24 \mathrm{~mm}$, are in good agreement with the experimental results. The dashed curves, which were calculated at large $s=72.4 \mathrm{~mm}$, fail to describe $f_{r 0}$, although the disagreement with $f_{z 0}$ is marginal. We note that our evaluation of $P_{\mathrm{ac}, 0}=1.35 \mathrm{kPa}-\mathrm{rms}$ was based on $s=7.24 \mathrm{~mm}$ (when the effect of finite $s$ is neglected, its value is $1.24 \mathrm{kPa}-\mathrm{rms}$ ). Although the influence of the droplet size was not considered in this theoretical analysis, a small but finite droplet-size dependence on the resonance frequencies was observed: when $d$ was decreased from 1.04 to $0.41 \mathrm{~mm}, f_{r 0}$ shifted from 6.1 to $6.35 \mathrm{~Hz}$ at $P_{\mathrm{ac}} / P_{\mathrm{ac}, 0}=1.6$.

Figure 3 (b) shows the relationship between the damping coefficient and droplet diameter for $P_{\mathrm{ac}} / P_{\mathrm{ac}, 0}$ in the range of 2-2.5. These

(a)

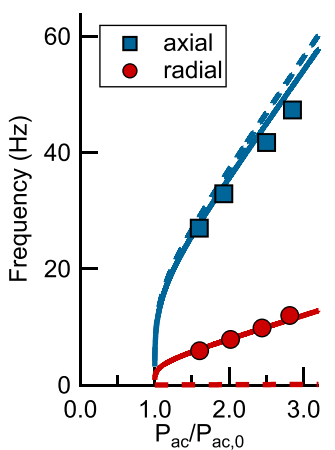

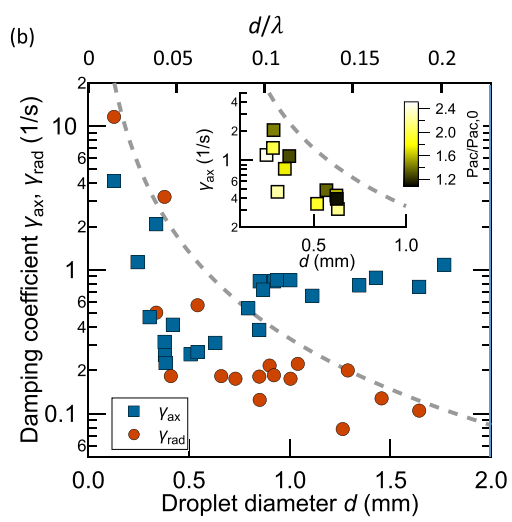

FIG. 3. (a) Comparison of theory and experiment (data points) for the axial and radial resonance frequencies. The solid curves and the dashed lines were calculated with $s=7.24$ and $72.4 \mathrm{~mm}$, respectively. (b) Relationship between droplet diameter $d$ and the damping coefficient measured at $P_{\mathrm{ac}} / P_{\mathrm{ac}, 0}$ in the range of 2-2.5. The inset shows the axial damping coefficients measured for $P_{\mathrm{ac}} / P_{\mathrm{ac}, 0}$ in the range of 1.2-2.4. The dashed curve shows the calculated damping coefficient expected from the Stokes coefficient.

values were evaluated from the frequency characteristics in Fig. 2 and from measurements of oscillation decays. We found that when $d$ was larger than $0.1-0.3 \mathrm{~mm}$, the oscillation decay was highly anisotropic, and the damping coefficients $\gamma_{\text {rad }}$ and $\gamma_{\text {ax }}$ in the radial and axial directions deviated from the $d^{-2}$ dependence expected from $\gamma_{s}$ (dashed curve). The values of $\gamma_{\mathrm{ax}}$ measured for $P_{\mathrm{ac}} / P_{\mathrm{ac}, 0}$ between 1.2 and 2.5 depicted in the inset show that the order-of-magnitude deviation between $\gamma_{\mathrm{ax}}$ and $\gamma_{\mathrm{s}}$ at $d \sim 0.3 \mathrm{~mm}$ at $P_{\mathrm{ac}} / P_{\mathrm{ac}, 0}=2.4$ was reduced to within a factor of $\sim 2$ at $P_{\mathrm{ac}} / P_{\mathrm{ac}, 0}=1.2$. No deformation of the droplet shape was observed during the oscillation decay, perhaps because the frequency range of the Rayleigh modes on the surface of the droplets was much higher than the $\gamma$ observed here. ${ }^{30}$

For large droplets, their anisotropic shape leads to an anisotropic damping coefficient. ${ }^{31}$ However, this effect is orders of magnitude smaller than the present observation: in Fig. 3, when $d=1.64 \mathrm{~mm}$, $d_{x}=1.74 \mathrm{~mm}$, and $d_{z}=1.42 \mathrm{~mm}$, we observed $\gamma_{\mathrm{ax}} / \gamma_{\mathrm{rad}}=6.91$. In contrast, the estimated anisotropy of the drag force ratio $\gamma_{S, a x} / \gamma_{S, \text { rad }}$ is equal to 1.05 for such an oblate spheroid in Stokes flow. ${ }^{31}$ The horn and reflector of the levitator may act as a wall to increase the drag force on droplets in the levitator cavity. ${ }^{32}$ However, this mechanism is not effective in our case: according to the experimental results reported by Lecoq et al., ${ }^{32}$ the estimated increase in $\gamma_{\text {rad }}$ is only $\sim 10 \%$ for droplets with $d \sim 1 \mathrm{~mm}$ and is even less for smaller droplets, and there is no effect on $\gamma_{\mathrm{ax}}$.

The upper panel of Fig. 4(a) shows the relationship between $\left(x_{c}\right.$, $\left.z_{c}\right)$ and $d=\left(d_{x}^{2} d_{z}\right)^{1 / 3}$ measured at $P_{\mathrm{ac}} / P_{\mathrm{ac}, 0}=2.2$. Figure $4(\mathrm{~b})$ shows the variations of $d_{x}$ and $d_{z}$ with time, and it can be seen that the difference between these diameters becomes smaller as the droplet size is reduced in time. The evaporation rate is proportional to the surface area, ${ }^{9}$ as can be seen from the linear decrease in droplet surface area also shown in Fig. 4(b). The smallest values of $d$ observed in these
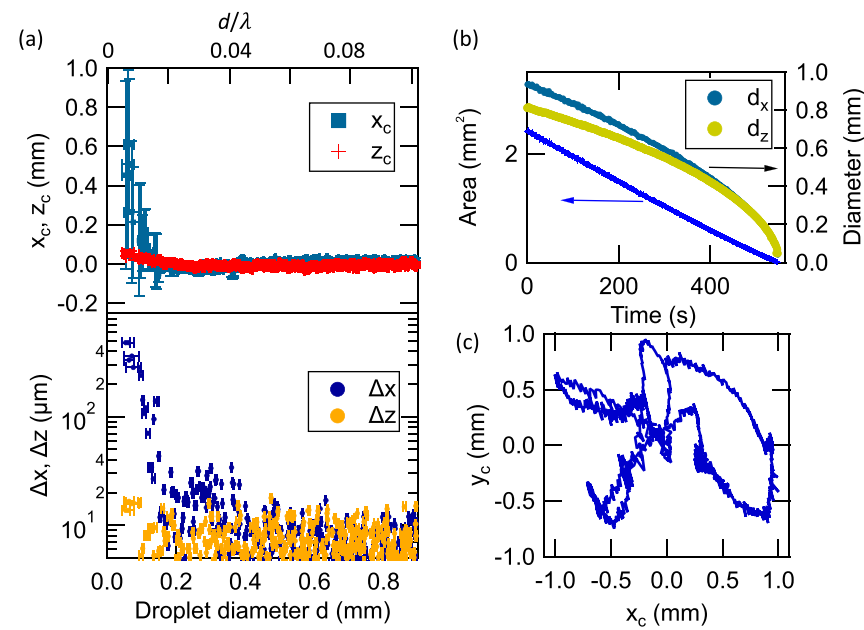

FIG. 4. (a) Relationship between droplet diameter $d$ and center-of-mass position $\left(x_{c}, Z_{c}\right)$ (upper panel) and the corresponding positional fluctuations $(\Delta x, \Delta z)$ in the radial $(x)$ and axial $(z)$ directions (lower panel), measured at $P_{a \mathrm{ac}} / P_{\mathrm{ac}, 0}=2.3 . d$ is defined as $\left(d_{x}^{2} d_{z}\right)^{1 / 3}$, where $d_{x}$ and $d_{z}$ are the droplet diameters in the $x$ and $z$ directions. $\Delta x$ and $\Delta z$ are also shown by the error bars for $x_{c}$ and $z_{c}$. (b) Variations of droplet surface area and of $d_{x}$ and $d_{z}$ in time. (c) Trajectory of a water droplet with $d=0.09 \mathrm{~mm}$ within the radial plane, measured at $P_{\mathrm{ad}} / P_{\mathrm{ac}, 0}=2.3$. The $y$ direction is in the radial plane, perpendicular to the $x$ direction. 
measurements were in the range of $0.05-0.1 \mathrm{~mm}$. Observation of smaller droplets was difficult because of the increased magnitude of the radial drift as well as the short evaporation time (a few seconds) of droplets of such sizes. The bottom panel of Fig. 4(a) shows the relationship between the positional fluctuations $(\Delta x, \Delta z)$ and $d$. These are also displayed as error bars for $x_{c}$ and $z_{c}$ in the upper panel.

These results show that for $d$ larger than $0.1-0.2 \mathrm{~mm}$, a positional stability of the droplet equal to $10-30 \mu \mathrm{m}$ is sufficiently small for precision applications such as the protein crystallography experiments that have recently been demonstrated. ${ }^{1,2}$ From the high-speed camera recording (see Fig. S4, supplementary material), we identified the observed $\Delta x$ and $\Delta z$ of $\sim 10 \mu \mathrm{m}$ of droplets with $d>0.2 \mathrm{~mm}$ as being due to excitation of the resonance oscillation (which persisted down to the smallest droplets in the axial direction).

When $d$ was in the range of $0.2-0.4 \mathrm{~mm}$, an increase in $\Delta x$ by 20-30 $\mu \mathrm{m}$ compared with the value at larger $d$ was observed. This was found to be an enhanced radial resonance oscillation. We were able to suppress it by reducing $P_{\mathrm{ac}} / P_{\mathrm{ac}, 0}$ to less than $\sim 2$ or to enhance it at higher pressures (see Fig. S5, supplementary material). The enhanced oscillation decayed when $d$ was reduced to $0.1-0.2 \mathrm{~mm}$, when $\gamma_{\text {rad }}$ increased by an order of magnitude as $d$ became smaller. Rudnick and Barmat $z^{33}$ studied the oscillatory instability of a levitated particle and proposed that the onset pressure for instability is reduced when the driving frequency is detuned from the resonance value of the air cavity of the levitator. We therefore repeated our measurements, but with the transducer-mirror distance varied instead of the ultrasound frequency. However, we observed no influence of detuning for our small levitated droplets at $P_{\mathrm{ac}} / P_{\mathrm{ac}, 0}=1.9$ when the transducer-mirror distance was shifted from the resonance by $+21 \%$ and $-27 \%$ of the full width at half maximum (equal to $1 \mathrm{~mm}$ for the fifth resonance of our levitator).

When $d$ was smaller than $\sim 0.2 \mathrm{~mm}, \Delta x$ increased from 10 to $30 \mu \mathrm{m}$ to $0.1-0.5 \mathrm{~mm}$, and $x_{c}$ drifted strongly in the range $\sim 1 \mathrm{~mm}$. This was found to be a random drift within the horizontal plane, as can be seen from the typical horizontal droplet trajectory in Fig. 4(c). This was obtained by capturing the motion of a droplet with $d=0.09 \mathrm{~mm}$ from above using a high-speed camera (at a frame rate of $125 \mathrm{~Hz}$ in a direction at an angle of $30^{\circ}$ from the horizontal plane). In contrast to the radial direction, positional stability of the droplet was retained in the axial direction down to the smallest sizes $(\sim 50 \mu \mathrm{m})$ measured in the experiment. We consider that this was because of the order-of-magnitude larger acoustic radiation force in the axial direction than the radial direction, given by the ratio $\left(f_{\mathrm{ax}} / f_{\mathrm{rad}}\right)^{2} \sim 16$.

The range $d \leqq 0.24 \mathrm{~mm}$ in which the droplet exhibited radial drift motion coincided with the range for which $\gamma_{\mathrm{rad}}$ and the drag force increased from an anomalously small amount to the order-of-magnitude larger values expected from the Stokes coefficient. Therefore, we tentatively ascribe the observed radial drift to the increased dominance of the drag force when $u_{f, r}$ is finite. $u_{f, r}$ may be enhanced for small droplets, especially when the droplet moves to a nonsymmetric position. As a numerical estimate, we compare the order-of-magnitude acoustic streaming velocity $V_{\text {st }}$ given by $V_{\text {ac }}^{2} / c_{0}$ (in the absence of the levitated droplets) with the radial flow velocity $u_{f, r}^{\text {expt }}$ from our experiment. The latter is estimated from Eqs. (1) and (2) as $\left(2 \pi f_{\mathrm{ac}}\right)^{2} \delta x_{c} / \gamma_{\mathrm{rad}}$, where $\delta x_{c}$ is the radial displacement and the radial velocity of the droplet $(\sim 0.5 \mathrm{~mm} / \mathrm{s})$ has been neglected. At $P_{\mathrm{ac}} / P_{\mathrm{ac}, 0}=2.3$ and $V_{\mathrm{ac}}=7.52 \mathrm{~m} / \mathrm{s}, \quad V_{\mathrm{st}}$ is equal to $0.16 \mathrm{~m} / \mathrm{s}$. From the observed $\gamma_{\mathrm{rad}}=11.6 \mathrm{~s}^{-1}$ for droplets with $d=0.1 \mathrm{~mm}$ at $P_{\mathrm{ac}} / P_{\mathrm{ac}, 0}=2$ and with $f_{\text {ac }} \sim 10 \mathrm{~Hz}$ and $\delta x_{c} \sim 0.5 \mathrm{~mm}$, we find that $u_{f, r}^{\text {expt }}$ is equal to $\sim 0.17 \mathrm{~m} / \mathrm{s}$. This order-of-magnitude agreement between $V_{\text {st }}$ and $u_{f, r}^{\text {expt }}$ is consistent with our interpretation above. Further quantitative analysis requires quantitative evaluation of the acoustic streaming around droplets $^{9}$ for such small values of $d$. However, this is beyond the scope of the present work. Nevertheless, a similar size dependence of the movement of a droplet or particle in an ultrasonic standing wave, that is, with large particles being strongly affected by the acoustic radiation force and small particles following the acoustic streaming, was reported in microfluidic channels when the density of the particles was close to the fluid density, ${ }^{34}$ as well as for water droplets in air. ${ }^{35}$ In the latter case, it was shown that $5 \mu \mathrm{m}$ water droplets could act as tracer particles for particle image velocimetry of acoustic streaming ${ }^{9,36-39}$ around levitated droplets.

The influence of viscosity on the acoustic radiation force has been studied recently. This influence is expected to increase as the droplet becomes smaller and its radius $d / 2$ becomes closer to the Stokes layer thickness $\delta=\sqrt{\nu /\left(\pi f_{\mathrm{ac}}\right)}$ (where $\nu$ is the kinetic viscosity, equal to $1.57 \times 10^{-5} \mathrm{~m}^{2} / \mathrm{s}$ for ambient air). ${ }^{40-43}$ Increased scattering of ultrasound by smaller droplets is expected, ${ }^{44,45}$ as well as increased translational movement of the droplets by incident acoustic waves via the visco-inertial effect. The temperature dependence of the dynamic viscosity is also expected to become more significant as the droplet size decreases. However, at $39 \mathrm{kHz}, \delta=11 \mu \mathrm{m}$ is still five times smaller than the radius of a droplet with $d=0.1 \mathrm{~mm}$. Therefore, it does not seem that the influence of the air viscosity alone can account for the observed radial drift of droplets with $d$ in the range of 0.1-0.2 $\mathrm{mm}$.

We note that the droplet stability will be affected by external air disturbances, although this can be avoided by enclosing the levitator as in the present experiment. The levitated droplet may also be disturbed by heating of the transducer and the resulting flow of warmed air: using a thermocouple, we observed that the transducer increased in temperature by up to $4-6^{\circ} \mathrm{C}$ for $P_{\mathrm{ac}} / P_{\mathrm{ac}, 0}$ in the range $2.3-2.6$, and random flow of the warmed air around the transducer could be seen in the schlieren imaging. There was a visible influence of this warmed air flow on levitated particles when the transducer was placed at the bottom of the levitator. However, when the transducer was placed at the top of the levitator, as in the present experiment, we were not able to detect any apparent correlation between the warmed air flow and droplet instabilities.

In summary, from a detailed study of the motion of water droplets in a single-axis acoustic levitator, we found that while the acoustic radiation force can be quantitatively described by inviscid fluid theory, a highly anisotropic and anomalous droplet-size dependence was observed for the damping coefficient, which deviated strongly from the values expected in the case of Stokes flow in free space. Positional stability of the levitated droplets in the range $10-30 \mu \mathrm{m}$ was demonstrated for droplets larger than $0.1-0.2 \mathrm{~mm}$. However, smaller droplets with $d / \lambda$ of 0.01 and below exhibited a random radial drift, which we ascribed to the increased drag force and the influence of acoustic streaming, even though their axial position stayed within $10-30 \mu \mathrm{m}$. Because of the convective flow around the levitated droplets induced by acoustic streaming, ${ }^{9,36-39}$ it seems plausible that the drag force acting on levitated droplets in an ultrasonic standing wave is very different from that in the case of Stokes flow in free space. Elucidation of the physical mechanism of the observed $d$ dependence of the damping coefficient will require further experiments on the effects of droplet 
density as well as further theoretical developments, but these are beyond the scope of the present letter.

The observed droplet-size dependence of the damping coefficients has consequences for applications that utilize the capture of ejected droplets as an automated method of sample loading, ${ }^{3}$ since the small damping coefficients limit the repetition rate of experiments. Because of their larger damping coefficients, droplets smaller than $0.1-0.2 \mathrm{~mm}$ allow a faster repetition rate. However, the resulting radial drift makes it difficult to utilize such small droplets. Further efforts to improve the positional stability of small droplets by controlling the acoustic streaming are under way, for example, by using a levitator with higher $f_{\mathrm{ac}}$ or with an inhomogeneous fluid as the levitating medium. ${ }^{46}$

See the supplementary material for a summary of the theory of the acoustic radiation force (Appendix A1), the estimation of the radial spread of the ultrasonic standing wave from the schlieren image (Appendix A2), representative oscillation decay data used to estimate the damping coefficients (Fig. S3), small oscillations of droplet positions (Fig. S4), results on positional stability measured at different ultrasound pressures (Fig. S5), and the schlieren image of the oscillating ultrasound pressure (supplementary material Video 1).

This work was partially supported by Innosuisse under Project No. 18726.1 in collaboration with leadXpro AG.

\section{REFERENCES}

${ }^{1}$ S. Tsujino and T. Tomizaki, Sci. Rep. 6, 25558 (2016).

${ }^{2}$ T. Tomizaki, A. Shinoda, and S. Tsujino, AIP Conf. Proc. 2054, 060072 (2019).

${ }^{3}$ S. Tsujino, A. Shinoda, and T. Tomizaki, Appl. Phys. Lett. 114, 213702 (2019).

${ }^{4}$ L. V. King, Proc. R. Soc. London, Ser. A 147, 212 (1934).

${ }^{5}$ L. P. Gorkov, Sov. Phys. Dokl. 6, 773 (1962).

${ }^{6}$ P. S. Larsen and J. Jensen, Int. J. Heat Mass Transfer 21, 511 (1978).

${ }^{7}$ M. Seaver, A. Galloway, and T. J. Manuccia, Rev. Sci. Instrum. 60, 3452 (1989).

${ }^{8}$ M. A. H. Weiser and R. E. Apfel, J. Acoust. Soc. Am. 71, 1261 (1982).

${ }^{9}$ A. Yarin, G. Brenn, O. Kastner, D. Rensink, and C. Tropea, J. Fluid Mech. 399, 151 (1999).

${ }^{10}$ E. H. Trinh, P. L. Marston, and J. L. Robey, J. Colloid Interface Sci. 124, 95 (1988).

${ }^{17}$ Y. J. Lü, W. J. Xie, and B. Wei, Appl. Phys. Lett. 87, 184107 (2005).

${ }^{12}$ D. Foresti, M. Nabaci, M. Klingauf, A. Ferrari, and D. Poulikakos, Proc. Natl. Acad. Sci. U. S. A. 110, 12549 (2013).

${ }^{13}$ A. Watanabe, K. Hasegawa, and Y. Abe, Sci. Rep. 8, 10221 (2018).

${ }^{14}$ S. Santesson and S. Nilsson, Anal. Bioanal. Chem. 378, 1704 (2004).
${ }^{15}$ J. Leiterer, F. Delißen, F. Emmerling, A. F. Thünemann, and U. Panne, Anal. Bioanal. Chem. 391, 1221 (2008).

${ }^{16}$ R. Tuckermann, L. Puskar, M. Zavabeti, R. Sekine, and D. McNaughton, Anal. Bioanal. Chem. 394, 1433 (2009).

${ }^{17}$ R. J. K. Weber, C. J. Benmore, S. K. Tumber, A. N. Tailor, C. A. Rey, L. S. Taylor, and S. R. Byrn, Eur. Biophys. J. 41, 397 (2012).

${ }^{18}$ A. Scheeline and R. L. Behrens, Biophys. Chem. 165-166, 1 (2012).

${ }^{19}$ T. Vasileiou, D. Foresti, A. Bayram, D. Poulikakos, and A. Ferrari, Sci. Rep. 6, 20023 (2016)

${ }^{20}$ E. H. Trinh, Rev. Sci. Instrum. 56, 2059 (1985).

${ }^{21}$ T. Fushimi, T. L. Hill, A. Marzo, and B. W. Drinkwater, Appl. Phys. Lett. 113, 034102 (2018).

${ }^{22}$ D. Foresti, M. Nabavi, and D. Poulikakos, J. Fluid Mech. 709, 581 (2012).

${ }^{23}$ M. A. B. Andrade, S. Polychronopoulos, G. Memoli, and A. Marzo, AIP Adv. 9, 035020 (2019).

${ }^{24}$ Z. Y. Hong, J. F. Yin, W. Zhai, N. Yan, W. L. Wang, J. Zhang, and B. W. Drinkwater, Sci. Rep. 7, 7093 (2017).

${ }^{25}$ D. L. Geng, W. J. Xie, N. Yan, and B. Wei, Appl. Phys. Lett. 105, 104101 (2014).

${ }^{26}$ L. D. Landau and E. M. Lifshitz, Fluid Mechanics (Pergamon, Oxford, 1987), p. 61.

${ }^{27}$ P. J. Holmes and D. A. Rand, J. Sound Vib. 44, 237 (1976).

${ }^{28}$ G. S. Settles, Schlieren Shadow Graph Techniques: Visualizing Phenomena in Transparent Media (Springer, Berlin, 2001), p. 25.

${ }^{29}$ V. N. Bindal, T. K. Saksena, S. K. Jain, and G. Singh, Appl. Acoust. 17, 125 (1984).

${ }^{30}$ L. Rayleigh, Proc. R. Soc. London, Ser. A 29, 71 (1879).

${ }^{31}$ F. Perrin, J. Phys. Radium 5, 497 (1934).

${ }^{32}$ N. Lecoq, F. Feuillebois, N. Anthore, R. Anthore, F. Bostel, and C. Petipas, Phys. Fluids A 5, 3 (1993).

${ }^{33}$ J. Rudnick and M. Barmatz, J. Acoust. Soc. Am. 87, 81 (1990).

${ }^{34}$ S. M. Hagsater, T. G. Jensen, H. Bruus, and J. P. Kutter, Lab Chip 7, 1336 (2007).

${ }^{35}$ K. Hasegawa, Y. Abe, and A. Goda, NPJ Microgravity 2, 16004 (2016).

${ }^{36}$ H. Schlichting, Phys. Z. 33, 327 (1932).

${ }^{37}$ E. H. Trinh and J. L. Robey, Phys. Fluids 6, 3567 (1994).

${ }^{38} \mathrm{~N}$. Riley, in Encyclopedia of Acoustics, edited by M. J. Crocker (Wiley, Hoboken, NJ, 1997), Vol. 1, p. 321.

${ }^{39}$ W. L. M. Nyborg, in Physical Acoustics, edited by W. P. Mason (Academic Press, Cambridge, MA, 1965), Vol. 2B, Chap. 11, p. 265.

${ }^{40}$ S. D. Danilov and M. A. Mironov, J. Acoust. Soc. Am. 107, 143 (2000).

${ }^{41} \mathrm{M}$. Settnes and H. Bruus, Phys. Rev. E 85, 016327 (2012).

${ }^{42}$ J. T. Karlsen and H. Bruus, Phys. Rev. E 92, 043010 (2015).

${ }^{43}$ R. E. Challis, M. J. W. Povey, M. L. Mather, and A. K. Holmes, Rep. Prog. Phys. 68, 1541 (2005).

${ }^{44}$ H. Lamb, Hydrodynamics, 6th ed. (Dover, New York, 1945), p. 659.

${ }^{45}$ R. J. Urick, J. Acoust. Soc. Am. 20, 283 (1948).

${ }^{46}$ J. T. Karlsen, W. Qiu, P. Augustsson, and H. Bruus, Phys. Rev. Lett. 120, 054501 (2018). 